\title{
VLBA images of high frequency peakers ${ }^{\star} \star \star \star$
}

\author{
M. Orienti ${ }^{1,2}$, D. Dallacasa ${ }^{1,2}$, S. Tinti ${ }^{3}$, and C. Stanghellini ${ }^{2}$ \\ 1 Dipartimento di Astronomia, Università di Bologna, via Ranzani 1, 40127 Bologna, Italy \\ e-mail: orienti@ira.inaf.it \\ 2 Istituto di Radioastronomia - CNR, via Gobetti 101, 40129 Bologna, Italy \\ 3 SISSA/ISAS, via Beirut 4, 34014 Trieste, Italy
}

Received 7 December 2005 / Accepted 11 January 2006

ABSTRACT

We propose a morphological classification based on the parsec scale structure of fifty-one High Frequency Peakers (HFPs) from the "bright" HFP sample. VLBA images at two adjacent frequencies (chosen among 8.4, 15.3, 22.2 and 43.2 GHz) have been used to investigate the morphological properties of the HFPs in the optically thin part of their spectrum. We confirm that there is quite a clear distinction between the pc-scale radio structure of galaxies and quasars: the $78 \%$ of the galaxies show a "Double/Triple" morphology, typical of Compact Symmetric Objects (CSOs), while the $87 \%$ of the quasars are characterised by Core-Jet or unresolved structure. This suggests that most HFP candidates identified with quasars are likely blazar objects in which a flaring self-absorbed component at the jet base was outshining the remainder of the source at the time of the selection based on the spectral shape.

Among the sources classified as CSOs or candidates it is possible to find extremely young radio sources with ages of about 100 years or even less.

Key words. galaxies: active - galaxies: nuclei - radio continuum: galaxies - galaxies: quasar: general

\section{Introduction}

Young radio sources, despite being very rare, are important tools in the study of the onset of radio emission. The radio plasma interacts with a presumably inhomogeneous and quite dense ambient medium in the vicinity of the active nucleus.

In the radio domain they have outstanding characteristics, being scaled down versions of the extended (up to a few Mpc) radio galaxies. Based on their radio morphology they are classified as "Compact Symmetric Objects" (CSOs), implying that they are intrinsically compact $(<1 \mathrm{kpc})$ and bright extragalactic radio sources $\left(P_{1.4 \mathrm{GHz}}>10^{25} \mathrm{~W} / \mathrm{Hz}\right)$ with a rather symmetric radio structure, and characterised by convex synchrotron radio spectra peaking at frequencies ranging from $100 \mathrm{MHz}$ to few GHz (Wilkinson et al. 1994). Both kinematic and spectral studies show that CSOs with sizes of 50-100 pc have typical ages of about $10^{3}$ years (Polatidis \& Conway 2003; Murgia 2003), still developing their radio emission within the host galaxy (Fanti et al. 1995; Readhead et al. 1996; Snellen et al. 2000).

\footnotetext{
* Tables 2-4 are only available in electronic form at the CDS via anonymous ftp to cdsarc.u-strasbg.fr $(130.79 .126 .5)$ or via http://cdsweb.u-strasbg.fr/cgi-bin/qcat?]/A+A/450/959

$\star \star$ Full Fig. 1 is only available in electronic form at http://www.edpsciences.org
}

The convex spectral shape is a distinctive property of the intrinsically small radio sources: there is an anti-correlation between the intrinsic peak frequency and the source size (e.g. O'Dea 1998) for the Compact Steep Spectrum sources of the Fanti et al. (1990) and the GHz-Peaked Spectrum of the Stanghellini et al. (1998) samples. It is explained in terms of synchrotron self-absorption occurring in a small radio emitting region. As the region (a mini radio lobe) expands as the result of the source growth, the turnover frequency moves to lower frequencies. It is also worth to mention that free-free absorption could play a role (e.g. Bicknell et al. 1997), more relevant in the smallest objects.

In this scenario the anti-correlation implies that the youngest objects have the highest turnover frequency, and therefore, sources with spectral peaks at frequencies higher than few $\mathrm{GHz}$ are good candidates to be newly born, with ages of about $10^{2-3}$ years.

A complete sample of "bright" radio sources with turnover frequency above $\sim 5 \mathrm{GHz}$, termed "High Frequency Peakers" (HFPs), has been selected by Dallacasa et al. (2000).

Since the selection of these sources is based on their simultaneous radio spectra at a single epoch, there is still some contamination from beamed radio sources, whose emission is temporarily dominated by a self-absorbed jet component.

Further simultaneous radio spectra from VLA observations (Tinti et al. 2005) showed that most of the sources identified 
with radio quasars modified the spectral shape due to flux density variability, and did not fulfill anymore the selection criteria. On the other hand most of the objects identified with galaxies (and empty field as well) preserved their convex radio spectra.

Another key aspect to understand these sources is related to their pc-scale morphology: it is well known that in young radio galaxies the radio emission is dominated by mini-hot-spots and mini-lobes. On the other hand, contaminating sources with temporarily peaked spectra should have core-jet structures. This paper reports on the results of new VLBA observations at two frequencies in the optically thin part of the spectrum of each source, chosen among 8.4, 15, 22 and $43 \mathrm{GHz}$ depending on the spectral peak frequency as found in Dallacasa et al. (2000).

Section 2 briefly recalls the sample selection criteria, Sect. 3 describes the observations and the production of the images, Sect. 4 presents the results, including the morphological classification, the spectral index distribution of the source components, and, finally, Sect. 5 summarises the main results from this work.

Throughout this paper, we assume $H_{0}=71 \mathrm{~km} \mathrm{~s}^{-1} \mathrm{Mpc}^{-1}$, $\Omega_{\mathrm{M}}=0.27$ and $\Omega_{\lambda}=0.73$. When redshift is unknown, we adopt $z=1.00$.

\section{The sample}

The bright HFP sample has been assembled by cross correlating the Green Bank survey (87GB) at $4.85 \mathrm{GHz}$ (Gregory et al. 1996) and the NRAO VLA Sky Survey (NVSS) at $1.4 \mathrm{GHz}$ (Condon et al. 1998): only the sources brighter than $300 \mathrm{mJy}$ at 4.85 GHz with inverted synchrotron spectra, and in particular those with a slope steeper than $-0.5\left(S \propto v^{-\alpha}\right)$ have been selected. Sources with $\left|b_{\text {II }}\right|<10^{\circ}$ have been excluded to avoid the galactic plane. Simultaneous VLA observations at eight different frequencies ranging from 1.365 to $22.46 \mathrm{GHz}$ were used to derive the radio spectrum and to remove variable sources from the sample.

The final sample of HFP candidates consists in 55 objects ( $\sim 3 \%$ of the total number of sources) with peaked radio spectra (Dallacasa et al. 2000), whose optical counterpart comprises: 11 galaxies, 33 quasars, $5 \mathrm{BL}$ Lac and 6 unidentified sources (Dallacasa et al. 2002, 2006). The redshift distribution is different between galaxies and quasars: typically galaxies redshift ranges between $\leq 0.1$ and 0.67 , while quasars, generally at higher redshift, between 0.9 and 3.6 (see Dallacasa 2003, for a recent review on the properties of this new class of objects).

\section{VLBA observations and data reduction}

Pc-scale resolution observations were carried out with the VLBA in 2002 (see Table 1) at 8.4, 15.3, 22.2 and 43.2 GHz, in full polarisation mode with a recording band-width of $32 \mathrm{MHz}$ at $128 \mathrm{Mbps}$. The correlation was performed at the VLBA correlator in Socorro.

The target sources, accommodated into four 9-h observing runs, were observed at a pair of frequencies in the optically thin region of their synchrotron spectra when this was possible: for
Table 1. Log of the VLBA observations.

\begin{tabular}{clcc}
\hline \hline Exp. Code & Date & Obs. time & Notes \\
\hline BD077A & 11 Jan. 2002 & $9 \mathrm{~h}$ & Erratic $T_{\text {sys }}$ values at LA \\
BD077B & 10 Feb. 2002 & $9 \mathrm{~h}$ & - \\
BD077C & 16 Feb. 2002 & $9 \mathrm{~h}$ & - \\
BD077D & 02 May 2002 & $9 \mathrm{~h}$ & - \\
\hline
\end{tabular}

example, the objects with turnover frequency above $15 \mathrm{GHz}$ in Dallacasa et al. (2000) were observed at 22.2 and 43.2 GHz.

Each target was observed typically for $20 / 30 \mathrm{~min}$ at each frequency, spread into 4 to 7 short scans at various HA in order to improve the uv coverage.

The strong sources 3C 454.3, 3C 345 and J2136+0041 (a "bright" HFP quasar) were used as fringe finders, and a few more calibration sources (including the "bright" HFP $\mathrm{J} 0927+3902$ and J1407+2827 alias OQ208) were observed to verify the system performance during each experiment.

All the data handling has been carried out by means of the NRAO AIPS package. A-priori amplitude calibration was derived using measurements of the system temperatures and the antenna gains. We estimate that the error on the absolute flux density scale is generally within $5 \%$, perhaps with a slightly larger value at $43.2 \mathrm{GHz}$.

Fringe fitting was carried out on each source, with a short (down to $1 \mathrm{~min}$ ) solution interval at 22.2 and $43.2 \mathrm{GHz}$ in order to preserve phase coherence. In general all sources were detected on all baselines, and the data turned out to have good quality at all frequencies, with the only exception of $\mathrm{J} 1526+6650$, which has not been detected at $43.2 \mathrm{GHz}$.

During the BD077A observations, the VLBA antenna located in Los Alamos was affected by technical problems that caused the system temperature have erratic values at 15.3 and $22.2 \mathrm{GHz}$, while the data were good at the other two frequencies.

The final radio images were obtained after a number of phase self-calibration iterations, and source parameters (total flux density and deconvolved sizes) have been generally measured by means of the task JMFIT for marginally resolved components, and with TVSTAT and IMSTAT when a component could not be fitted with a Gaussian profile. In cases where multiple components are present, the VLBA total flux density has been derived on the image plane by means of TVSTAT. All the values are reported in Tables $2-4$.

\section{Results}

Low dynamic range images are not the ideal tool to perform a very accurate morphological classification, and this is particularly true for complex sources. However, from our short observations it is possible to derive indications on the nature using also the spectral information allowed by the frequency pair data available for each source.

In our VLBA images fourteen out of the 51 sources we observed, show a CSO-like morphology, six present a Core-Jet structure and one has multiple images, being a well known gravitational lens (J1424+2256). These sources will be described in detail in Sect. 4.2. 
Despite the high resolution achieved by our VLBA images, about 30 sources $(\sim 60 \%)$ are still unresolved or marginally resolved. We consider "Marginally Resolved" those sources whose largest angular size (LAS) we detect is between 0.5 and 1 beam size at the highest frequency, and we term "Unresolved" all the sources whose LAS is smaller than half the beam size at both frequency. We then classify as Unresolved 25 objects ( 2 galaxies, 17 quasars, 3 BL Lacs, and 3 empty field), and "Marginally Resolved" 6 sources (all quasars). The total spectral index of unresolved and marginally resolved sources are usually quite flat or inverted, despite we are observing the optically thin emission of each source as from the VLA total spectra in Dallacasa et al. (2000).

If we compare the flux densities in our VLBA data, substantial variability has been found in two "Unresolved" and one "Marginally Resolved" objects. These sources, the quasars $\mathrm{J} 0722+3722$ and $\mathrm{J} 2212+2355$, and the BL Lac object $\mathrm{J} 1457+0749$, show an increment of about $20 \%$ and $10 \%$ in their flux density at the lower and higher frequencies, respectively. Therefore, they are likely flat spectrum sources seen in different phase of their variability, and happened to be classified as HFP radio sources since at the time of the simultaneous VLA multi-frequency observation their emission was dominated by a bright self-absorbed component peaking at $\mathrm{cm}$-wavelengths. As in Tinti et al. (2005), we point out these objects are not to be considered HFPs anymore.

\subsection{Source images}

Full resolution images of the fourteen sources with a CSO-like morphology are presented in Figs. 1 and 2. Figures 3 and 4 instead show images of the objects with a Core-Jet structure. An example of a "Marginally Resolved" source is shown in Fig. 5. Finally, Fig. 6 show the multiple images of the gravitational lens. In general the sources are presented in increasing RA order and images at the two frequencies are next to each other, the lowest frequency always on the left panel (Figs. 1 and 3). For the objects resolved at one frequency only, such image is shown (Figs. 2 and 4). Images for the objects found to be unresolved at both frequencies are not presented.

Noises in the image plane are generally between 0.5 and $1 \mathrm{mJy}$, except for few cases where bad weather conditions affected the observations implying substantially higher noise levels, particularly at high frequency.

For each image we provide the following information on the plot itself:

a) the source name and the observing frequency on the top left corner;

b) the peak flux density in $\mathrm{mJy} / \mathrm{beam}$;

c) the first contour intensity (f.c. in mJy/beam), which is generally 3 times the off-source rms noise level measured on the image; contour levels increase by a factor 2 ;

d) the optical identification;

e) The restoring beam, plotted on the bottom left corner of each image.
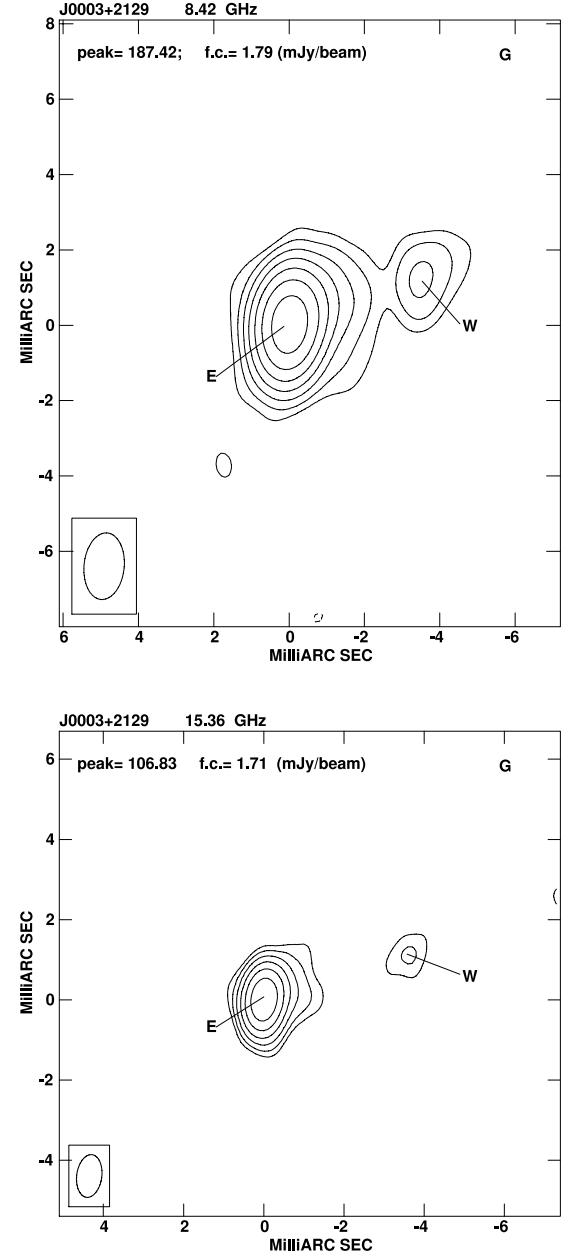

Fig. 1. An example of VLBA images at the two frequencies of a candidate HFP with a CSO morphology. For each image we give the following information on the plot itself: a) peak flux density in $\mathrm{mJy} /$ beam; b) first contour intensity (f.c., in mJy/beam), which is generally 3 times the rms noise on the image plane; contour levels increase by a factor of 2 ; c) the optical identification; d) the restoring beam is plotted on the bottom left corner of each image. All the images of Fig. 1 are available in electronic form at www. edpsciences.org.

\subsection{Comments on individual sources}

In this section we discuss in detail the properties of the sources which show a CSO-like or Core-Jet morphology, in the light of Figs. 1-4.

We note that the spectral index of the source components has been computed considering the full-resolution images at both frequencies, since they allowed the lowest rms noise levels in the images. This may produce an artificial steepening of the spectral index of the components, at least a few beam size wide. We note, however, that the vast majority of the sources and/or components are unresolved or marginally resolved, and the artificial steepening may occur in a very few cases.

Typical errors on the determination of the spectral index are of the order of 0.1 . 

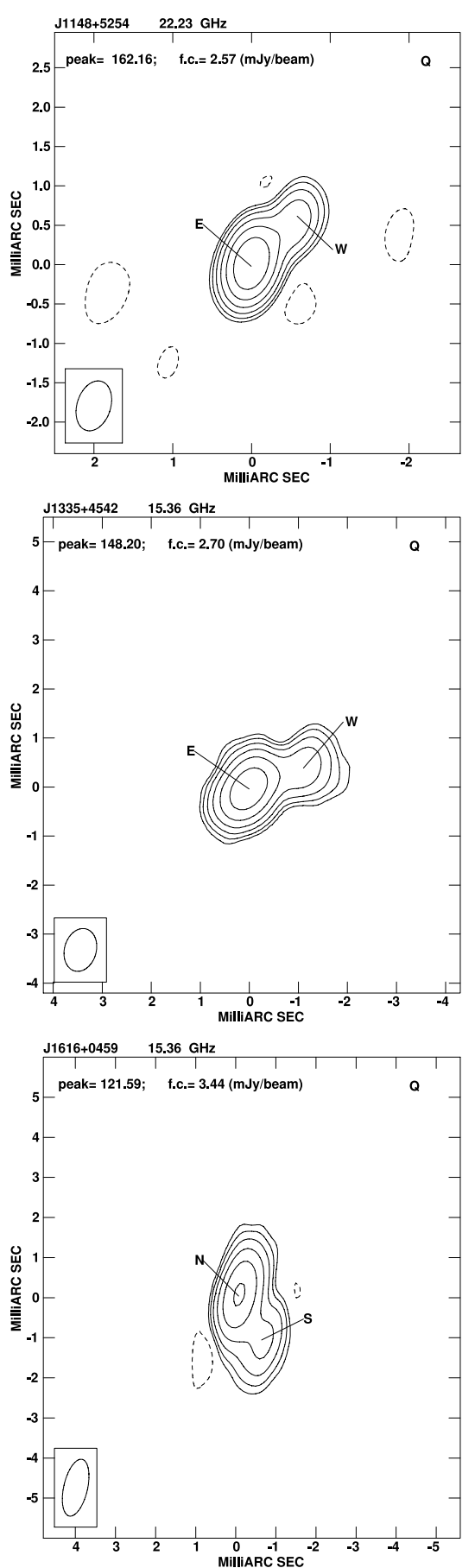

Fig. 2. VLBA images of the sources with a CSO-like morphology, which are resolved at highest frequency only. For each image we give the following information on the plot itself: a) peak flux density in $\mathrm{mJy} /$ beam; b) first contour intensity (f.c., in $\mathrm{mJy} /$ beam), which is generally 3 times the rms noise on the image plane; contour levels increase by a factor of 2 ; c) the optical identification; d) the restoring beam is plotted on the bottom left corner of each image.

\subsubsection{CSO candidates}

We consider CSO candidates those sources which have a "Double/Triple" morphology in our VLBA observations (Figs. 1, 2), an optically thin total spectral index $>0.5(S \propto$ $v^{-\alpha}$; Table 2), and small value of the variability index, as described in Tinti et al. (2005). Moreover, each source component is characterised by steep spectral indices (Table 3), with the only exception of the sources J0005+0524, J1335+5844 and $\mathrm{J} 1735+5049$, and our classification will be explained in more detail in the following discussion.

- J0003+2129: galaxy at $z=0.45$ (Dallacasa et al. 2006). This source appears as a very asymmetric Double, with flux density ratio of $\sim 25: 1$ at both frequencies. Mostly of the flux density originates within the Eastern component, while a weak feature, accounting for 8 and $5 \mathrm{mJy}$ at 8.4 and $15 \mathrm{GHz}$ respectively, is present to the West, $\sim 4$ mas apart. The total linear size (LS) is about 20 pc.

- J0005+0524: quasar at $z=1.887$. The radio emission originates within two well-resolved components, separated by 2 mas. Their flux density is almost the same at $8.4 \mathrm{GHz}$, while it increases significantly at $15 \mathrm{GHz}$ where $S_{\mathrm{W}} / S_{\mathrm{E}} \sim 3.6$. This source does not match one of our selection criteria. Indeed, the spectral index of the Western component is quite flat $\left(\alpha_{8.4}^{15} \sim\right.$ $0.2)$, while the other structure has a steep spectrum $\left(\alpha_{8.4}^{15} \sim 1.9\right.$; Table 3). Although these values, we still classify this source as a CSO candidate, since the flattish spectrum of the component W could be due either to a core component, or a very compact hot-spot. New observations with higher dynamic range and resolution are necessary to unambiguously classify this source.

- J0037+0524: this source has been found as an empty field by Dallacasa et al. (2002). It shows a Double morphology and the radio emission originates within two well-resolved components, $\sim 2$ mas apart. Their flux density ratio is $S_{\mathrm{E}} / S_{\mathrm{W}} \sim 5$ and 10 at 8.4 and $15 \mathrm{GHz}$ respectively.

- J0428+3259: galaxy at $z=0.479$ (Dallacasa et al. 2002, 2006). The images presented in Fig. 1 suggest that this source is an asymmetric Triple. The Eastern and the Western components are 2 mas and 1 mas respectively, from the central component, which is also the brightest one. Their flux density ratio is $S_{\mathrm{E}}: S_{\mathrm{W}}: S_{\mathrm{Ce}}=1: 4: 10$ and $1: 3.3: 14$, at 15 and $22 \mathrm{GHz}$, respectively. On the arcsecond-scale, at 1.4, 1.7 and 5.0 GHz it shows an extended emission accounting for 12,8 and $3 \mathrm{mJy}$ respectively, and with the same position angle of the VLBA structure (Tinti et al. 2005).

- J0638+5933: optically unidentified. This radio source can be classified as a Triple. The main component is marginally resolved in two different regions, with a flux density ratio of $S_{S 1} / S_{S 2} \sim 2$ and 4 at 22 and $43 \mathrm{GHz}$ respectively. Since the two components labelled as $\mathrm{N}$ and $\mathrm{N} 1$ are a single unresolved component at $22 \mathrm{GHz}$, when we compute the spectral index we must take into account the sum of their flux density at $43 \mathrm{GHz}$, obtaining $\alpha_{22}^{43} 0.4$, similar to the spectral index of the Southern component $(\sim 0.4)$. The similarity of the spectral indices, together with the fact that values of 0.4 are usually found in hotspot region, lead us to still classify this source as a CSO candidate, although it does not match our selection criteria. New high-resolution observations with a higher dynamic range are necessary to unambiguously classify this source.

About $11 \%$ of the total flux density is missing in our VLBA observations at $22 \mathrm{GHz}$.

- J0650+6001: quasar at $z=0.455$. This source has been confirmed as a CSO by Polatidis et al. (1999). The radio emission originates within two components, $\sim 3$ mas $(\sim 17$ pc) apart. A weak feature, visible only at $15 \mathrm{GHz}$ (Fig. 1) and accounting 

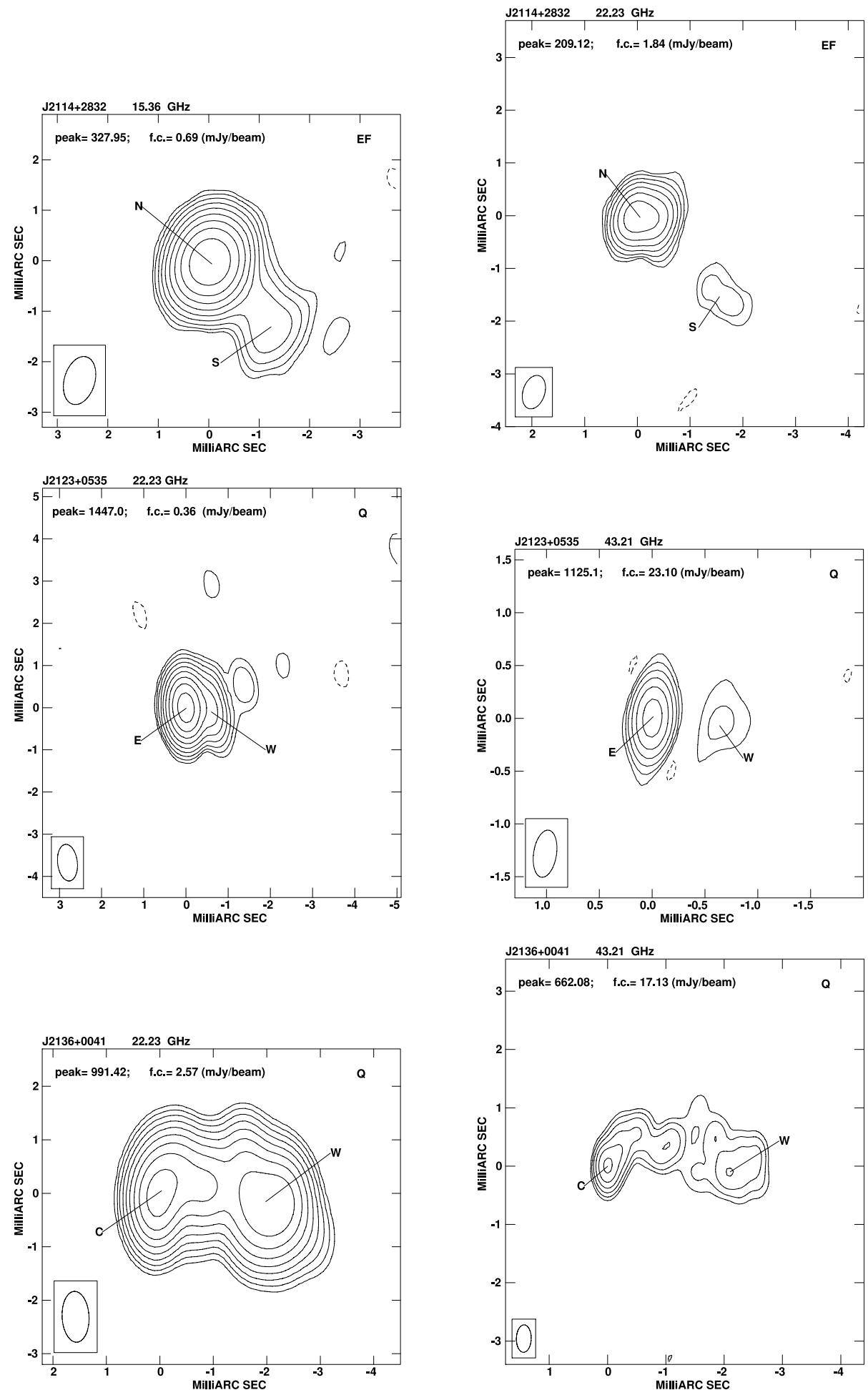

Fig. 3. VLBA images at the two frequencies of the sources with a Core-Jet morphology. For each image we give the following information on the plot itself: a) peak flux density in $\mathrm{mJy} /$ beam; b) first contour intensity (f.c., in $\mathrm{mJy} /$ beam), which is generally 3 times the rms noise on the image plane; contour levels increase by a factor of 2 ; c) the optical identification; d) the restoring beam is plotted on the bottom left corner of each image.

for $8 \mathrm{mJy}$, is present to the North of the Northern component, in agreement with other works at lower frequencies (Stanghellini et al. 1999). The Northern component (labelled as N) is compact and the brightest one, while the Southern is clearly resolved N-S. Their flux density ratio $S_{\mathrm{N}} / S_{\mathrm{S}}$ is $\sim 3.5$ at both frequencies. About $18 \%$ of the total flux density is missing in our VLBA image at $22 \mathrm{GHz}$. We note that in Tinti et al. (2005) the source shows some variability in flux density at high frequencies, although the spectral shape remains convex. 

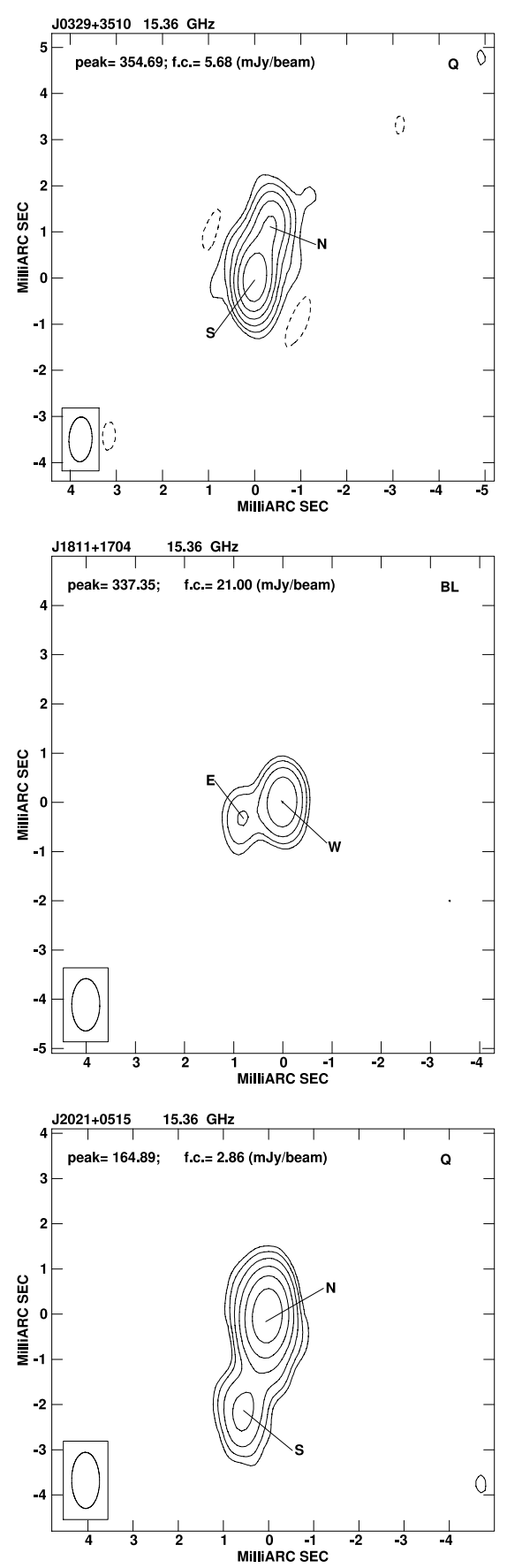

Fig. 4. VLBA images of the sources with a Core-Jet morphology, which are resolved at highest frequency only. For each image we give the following information on the plot itself: a) peak flux density in $\mathrm{mJy} /$ beam; b) first contour intensity (f.c., in $\mathrm{mJy} /$ beam), which is generally 3 times the rms noise on the image plane; contour levels increase by a factor of 2 ; $\mathbf{c}$ ) the optical identification; d) the restoring beam is plotted on the bottom left corner of each image.

- J1148+5254: quasar at $z=1.632$. Although at $15 \mathrm{GHz}$, this source appears only marginally resolved in the NW direction, at $22 \mathrm{GHz}$ (Fig. 2) it is clearly separated in two different asymmetric components. Their flux density ratio accounts for $S_{\mathrm{E}} / S_{\mathrm{W}} \sim 5.8$ and 4.7 at 15 and $22 \mathrm{GHz}$ respectively. We note that, although both components have steep spectra, the faintest is the flattest as well, with $\alpha_{15}^{22} \sim 0.6$, instead of 1.2 shown by

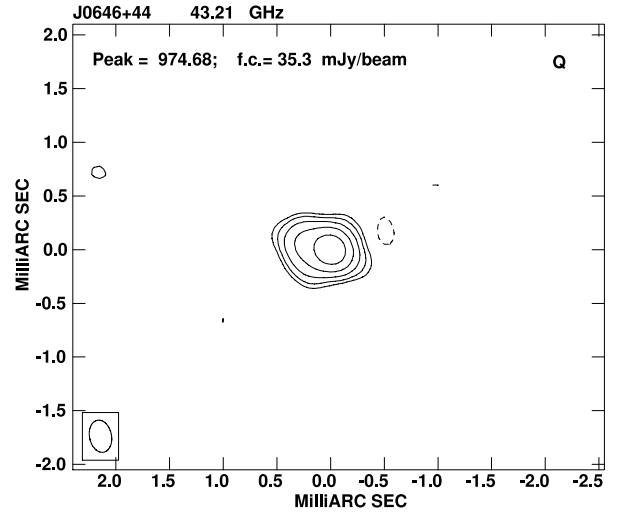

Fig. 5. VLBA image at $43 \mathrm{GHz}$ of the Marginally Resolved source J0646+4451. We give the following information on the plot itself: a) peak flux density in $\mathrm{mJy} / \mathrm{beam}$; b) first contour intensity (f.c., in $\mathrm{mJy} / \mathrm{beam}$ ), which is 3 times the rms noise on the image plane; contour levels increase by a factor of $2 ; \mathbf{c}$ ) the optical identification; d) the restoring beam is plotted on the bottom left corner.

the brightest. About $29 \%$ and $48 \%$ of the total flux density is missing in our VLBA images at 15 and $22 \mathrm{GHz}$ respectively, and this may complicate our interpretation further.

No information on the flux density variability is currently available given that this source was not observed by Tinti et al. (2005). The VLA observations considered here as a comparison with the VLBA total flux densities were carried out by Dallacasa et al. (2000), therefore it is possible that some amount of missing flux density could arise from intrinsic variability.

- J1335+4542: quasar at $z=2.449$. At $15 \mathrm{GHz}$ (Fig. 2), the radio emission clearly originates within two different components, while at the lower frequency the source appears marginally resolved in the NW direction only. Their flux density ratio is $S_{\mathrm{E}} / S_{\mathrm{W}} \sim 4$ at $15 \mathrm{GHz}$. The Eastern component has a spectral index $\alpha_{8.4}^{15} \sim 0.6$, while the other component has a very steep spectrum $\left(\alpha_{8.4}^{15} \sim 1.9\right.$; Table 3$)$, although the fit to the images, from which the flux densities were derived, proved to be rather problematic.

About $11 \%$ and $14 \%$ of the total flux density are missing in our VLBA images at 8.4 and $15 \mathrm{GHz}$, respectively.

- J1335+5844: there is an optical identification with a very weak object (likely a galaxy) reported by Dallacasa, Falomo \& Stanghellini (2006). The optically thin spectral index of this source is 0.5 . At $8.4 \mathrm{GHz}$ the source is clearly resolved into two compact components in agreement with what found by Xiang et al. (2002), while at $15 \mathrm{GHz}$ the Southernmost component becomes weak and extended. As shown in Fig. 1, the two components are separated by $\sim 15$ mas. Their flux density ratio is $S_{\mathrm{N}} / S_{\mathrm{S}} \sim 3$ and 8 at 8.4 and $15 \mathrm{GHz}$ respectively. This source does not match one of our selection criteria. In fact, the Northern has a flattish spectrum $\left(\alpha_{8.4}^{15} \sim 0.1\right)$, while the spectral index of the Southern component is very steep $>2$ (Table 3 ). For these reason, Peak \& Taylor (2000) did not consider this source as a CSO, and it was not included in the COINS sample. However, we still classify this source as a CSO candidate, since the flattish spectrum of the Northern component could be due 

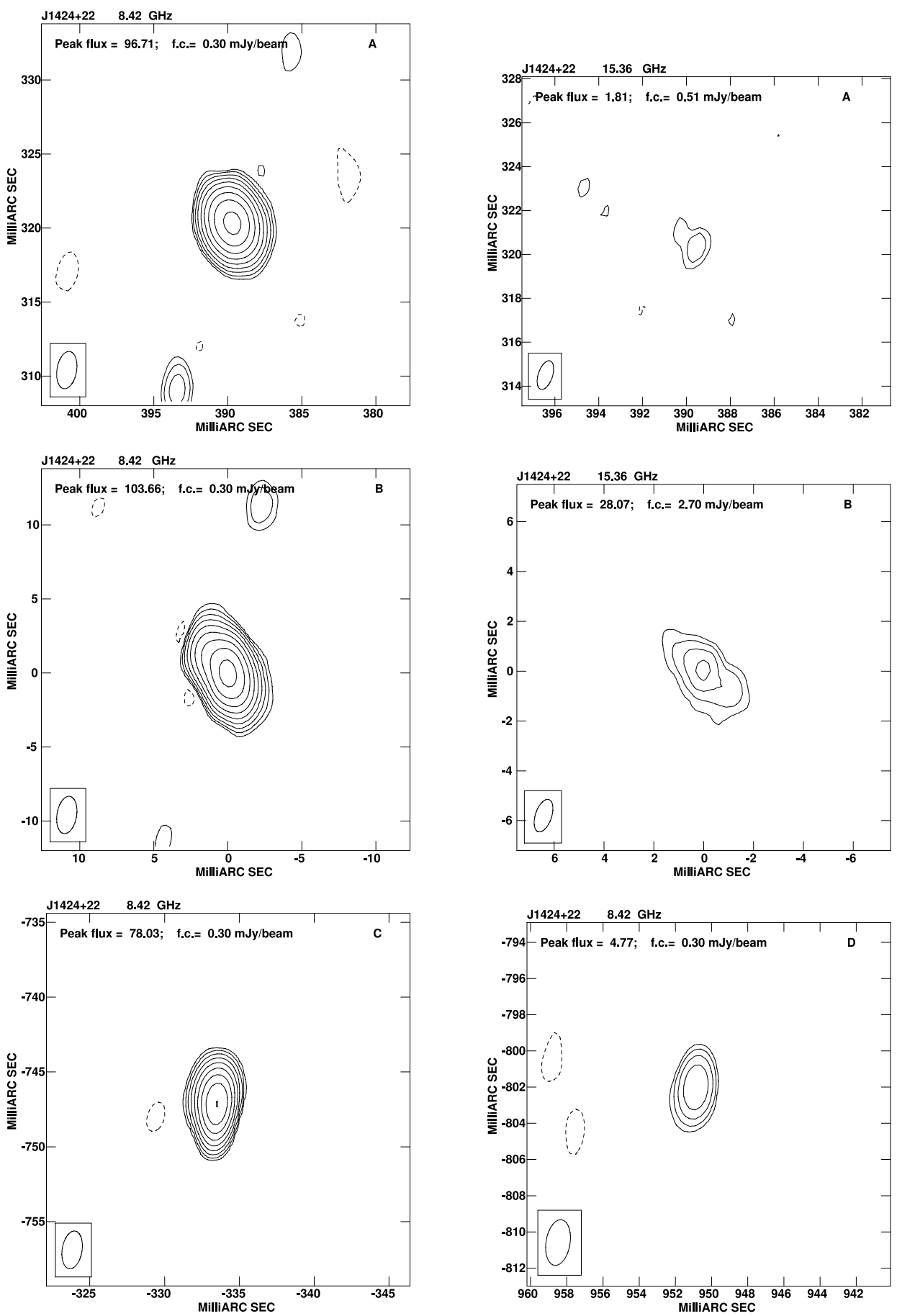

Fig. 6. VLBA images of the gravitational lens system J1424+2256. Top: images at 8.4 and $15 \mathrm{GHz}$ of Component A. Middle: images at 8.4 and $15 \mathrm{GHz}$ of Component B. Bottom: images at 8.4 of Component C (left) and D (right). For each image we give the following information on the plot itself: a) peak flux density in $\mathrm{mJy} / \mathrm{beam}$; b) first contour intensity (f.c., in mJy/beam), which is generally 3 times the rms noise on the image plane; contour levels increase by a factor of 2; c) the restoring beam is plotted on the bottom left corner of each image.

to either an embedded core component, or a very compact hotspot where self-absorption is relevant (Xiang et al., in preparation). Indeed, in VLBI images with a dynamic range higher than those presented here, it has been possible to identify the core component of this source, which is located about midway from the outer and much brighter components (Dallacasa et al. 2005). About $10 \%$ and $15 \%$ of the total flux density is missing in our VLBA images at 8.4 and $15 \mathrm{GHz}$ respectively.
- J1511+0518: it is optically identified with a Seyfert galaxy at redshift 0.081 (Chavushyan et al. 2001). The optically thin spectral index of the source is very steep with $\alpha_{15}^{22} \sim 1.2$.

At $15 \mathrm{GHz}$ it is clearly resolved in three well-separated, aligned components. The flux density of the outer components is $S_{\mathrm{E}} / S_{\mathrm{W}} \sim 2$ at both frequencies. On the other hand, the inner component accounts for 14 and $4 \mathrm{mJy}$ at 15 and $22 \mathrm{GHz}$ respectively, indicating a very steep spectral index $\alpha_{15}^{22}>3$. 
About $30 \%$ of the total flux density is missing in our VLBA images at both frequencies.

- J1616+0459: quasar at $z=3.197$. At $8.4 \mathrm{GHz}$ this source consists of a bright component, labelled as $\mathrm{N}$, and a slightly resolved structure in the SW direction ( $S$ component), while at $15 \mathrm{GHz}$ the radio emission clearly originates within two different components, $\sim 1.4$ mas (11 pc) apart (Fig. 2). Their flux density ratio is $S_{\mathrm{N}} / S_{\mathrm{S}} \sim 3.5$ at both frequencies. About $11 \%$ and $21 \%$ of the total flux density is missing in our VLBA images at 8.4 and $15 \mathrm{GHz}$ respectively.

- J1735+5049: optically identified with a very faint object, tentatively classified as a galaxy by Stickel \& Kühr (1996). The optically thin spectral index of this source is $<0.5$. This source shows a Double morphology, in agreement with what found by Xiang et al. (2002). As for J1335+5844, it does not match our selection criteria, since the Northern component is characterised by a steep spectrum $\alpha_{8.4}^{15} \sim 1.2$, while the Southern component has a flattish spectrum of about 0.3 . For this reason, Peck \& Taylor (2000) did not consider this source as a $\mathrm{CSO}$, and it was not included in the COINS sample. However, we still classify this source as a CSO candidate, since the flattish spectrum of the Southern component could be due to either a core component, or a very compact hot-spot. New highresolution observations are necessary to unambiguously classify this source. About $9 \%$ of the total flux density is missing in our VLBA image at $15 \mathrm{GHz}$.

- 1855+3742: optically identified with a galaxy (Dallacasa et al. 2006). The pc-scale structure is quite different from all the other sources discussed so far, presenting an elongated structure which is well resolved in the $15 \mathrm{GHz}$ image. At $8.4 \mathrm{GHz}$ the flux density is clearly peaked on the source centre, while at $15 \mathrm{GHz}$ is more uniformly distributed. About $14 \%$ and $33 \%$ of the total flux density is missing in our VLBA observations at 8.4 and $15 \mathrm{GHz}$, respectively.

- J2203+1007: weak galaxy with unknown redshift (Dallacasa et al. 2002). This source has been confirmed as a CSO by Gugliucci et al. (2005). It shows a Triple morphology, but the radio emission mainly originates in the outer components, located about 10 mas apart. Their flux density ratio is $S_{\mathrm{E}} / S_{\mathrm{W}} \sim$ 2.2 and 4.8 at 8.4 and $15 \mathrm{GHz}$ respectively. As in J1511+0518, the central component, which accounts for $11 \mathrm{mJy}$ at $8.4 \mathrm{GHz}$, is completely resolved out at $15 \mathrm{GHz}$, indicating a very steep spectral index. About $19 \%$ of the total flux density is missing in our VLBA image at $15 \mathrm{GHz}$.

\subsubsection{Core-Jet sources}

Here we describe the properties of the six Core-Jet sources. We consider Core-Jet those sources which have a compact component (containing the source core) with a flat spectrum, and the other structures with a steeper spectrum (Table 4).

- J0329+3510: quasar at $z=0.50$ (Dallacasa et al. 2006). The pc-scale radio structure of this source is characterised by a compact and bright component, and a second one located at $\sim 1$ mas (7 pc) apart in the NW direction (Fig. 4). Their flux density ratio is $S_{\mathrm{S}} / S_{\mathrm{N}} \sim 2$ at both frequencies and both the radio components have an inverted spectral index $\left(\alpha_{8.4}^{15} \sim-0.2\right.$; Table 4). Extended emission have been detected on the arcsecond-scale with the VLA at $1.4,1.7$ and $4.9 \mathrm{GHz}$ (Tinti et al. 2005). In the VLA second epoch observations it no longer shows the convex spectrum, leading us to the conclusion that this is a blazar object, and it will be rejected from the sample of genuine young and small radio sources.

- J1811+1704: optically identified with a stellar object by Dallacasa et al. (2002). Its optical spectrum has been found featureless (Dallacasa et al. 2006), and then we can consider it as a BL Lac object.

This source is characterised by a Double morphology at the highest frequency, while it is only marginally resolved in the E direction at $8.4 \mathrm{GHz}$. The brightest component has an inverted spectrum $\left(\alpha_{8.4}^{15} \sim-0.1\right)$, indicating the presence of the source core. It shows an arcsecond-scale structure in the VLA images at 1.4 and $1.7 \mathrm{GHz}$ (Tinti et al. 2005). In the VLA second epoch observations it no longer shows the convex spectrum, leading us to confirm it as a blazar object, and then it will be rejected from the sample of young and small radio sources. - J2021+0515: optically identified with a galaxy by Dallacasa et al. (2002). At $15 \mathrm{GHz}$, the radio emission originates within two well-resolved components, $\sim 2$ mas apart, while at $8.4 \mathrm{GHz}$ it appears Marginally Resolved. Super-resolving the lowerfrequency image in the $\mathrm{N}-\mathrm{S}$ direction, indicates that the overall structure might be an asymmetric double. Although the radio emission mainly originates within the brightest component, labelled as $\mathrm{N}$ in Fig. 2, its spectrum is steeper than the Southern component $\left(\alpha_{8.4}^{15} \sim 0.8\right.$ and -0.4 for $\mathrm{N}$ and $\mathrm{S}$ component, respectively). About $10 \%$ of the total flux density is missing in our VLBA image at $15 \mathrm{GHz}$.

- J2114+2832: optically identified with an unresolved object with $R=18.35$ (Dallacasa et al. 2002). This source is characterised by a compact and bright component, which accounts for $93 \%$ of the total flux density and with a spectral index of $\alpha_{15}^{22} \sim 0.6$. At $22 \mathrm{GHz}$ this component is marginally resolved in the E-W direction, suggesting that we are looking at the jetbase. Another extended low-brightness structure with a steep spectral index $\left(\alpha_{15}^{22} \sim 1\right)$ is located $\sim 2$ mas apart. About $39 \%$ and $47 \%$ of the total flux density is missing in our VLBA images at 15 and $22 \mathrm{GHz}$, respectively. It should be noted that this source has not been observed by Tinti et al. (2005). The VLA observations considered as a comparison between the total flux densities were carried out by Dallacasa et al. (2000), therefore it is likely that intrinsic variability may account for some amount of missing flux density.

- J2123+0535: quasar at $z=1.878$. The radio emission of this source mainly originates within a compact region (E component) which accounts for $\sim 90 \%$ of the total flux density. A weak feature, visible in the VLBA image at $43 \mathrm{GHz}$ and only marginally resolved at the lower frequency, is present to the Western part of the main component, so the radio morphology is that of a Double. Both spectral indices are quite flat ( $\alpha_{22}^{43} \sim 0.3$ and 0.5 for the $\mathrm{E}$ and $\mathrm{W}$ component, respectively). About $33 \%$ of the total flux density is missing in our VLBA image at $22 \mathrm{GHz}$. This source was a GPS candidate, observed by the ATCA monitoring program. Although its convex spectrum, it has been rejected as new GPS candidate for the evidence of significant variability (Edwards et al. 2004). In the 
second-epoch VLA observations it no longer shows the convex spectrum, leading us the conclusion that this is a blazar object, and it will be dropped from the sample.

$-\mathrm{J} 2136+0041$ : quasar at $z=1.932$. This object was recognised early as a GPS source (Shimmins et al. 1968). It has a CoreJet morphology, although at low frequency it may appears as a Double with components of similar flux density. The source core is located in the Easternmost component (labelled as C), while the jet is initially directed to the North and then turns to the West. The spectral index of the jet is rather steep $\alpha_{22}^{43} \sim$ 1.3 , while the core has a flatter spectrum $\left(\alpha_{22}^{43} \sim 0.7\right)$, although this value may be slightly increased by some emission from the steep-spectrum jet-base. It has been noted that, although this source presents some amount of flux density variability, its GPS spectral shape does not change with time (Tornikoski et al. 2001). About $57 \%$ of the total flux density is missing in our VLBA image at $22 \mathrm{GHz}$. The VLA observations considered as a comparison between the total flux densities were carried out by Dallacasa et al. (2000), therefore it is possible that some amount of missing flux density could be related to an intrinsic variability.

\subsubsection{The gravitational lens J1424+2256}

The gravitational lens $\mathrm{J} 1424+2256$ is associated with a quasar of 16.5 magnitude at $z=3.62$. For this reason is one of the highest apparent luminosity lens system (Patnaik et al. 1992). The lensing object belong to a group of galaxies at $z=0.338$ (Kudnic et al. 1997).

In our VLBA observations the lens system consists of four components (Fig. 6). Considering the position angle of the three brightest components, $\mathrm{PA}_{\mathrm{A}} 56^{\circ}, \mathrm{PA}_{\mathrm{B}} 43^{\circ}$ and $\mathrm{PA}_{\mathrm{C}} 14^{\circ}$, they are tangentially elongated as expected from lens models. Component $\mathrm{D}$ is unresolved in our observations. Their flux densities at $8.4 \mathrm{GHz}$, accounting for $S_{\mathrm{A}} 156 \mathrm{mJy}, S_{\mathrm{B}} 169 \mathrm{mJy}$, $S_{\mathrm{C}} 89 \mathrm{mJy}$ and $S_{\mathrm{D}} 4.6 \mathrm{mJy}$, as well as their position angles, are in agreement with what found by Patnaik et al. (1999). At $15 \mathrm{GHz}$, only the two brightest components have been detected. Component B shows the same elongation as at lower frequency, while component $\mathrm{A}$ is almost resolved out.

From VLA and VLBI observations some amount of polarised emission have been found in the three brightest components, accounting for $2.5 \%, 1.8 \%$ and $1.2 \%$ in A, B and C respectively. Component $\mathrm{D}$ was too faint to detect any polarisation (Patnaik et al. 1999, 1992).

\section{Discussion}

Here we present an overview of the properties derived from the pc-scale structure as seen in our observations. A detailed discussion on the physical properties will be given in a forthcoming paper.

\subsection{Morphology}

The search for young radio sources based only on their spectral shape has proven successful, although there is some amount of contamination by flat-spectrum, variable objects caught when their radio emission is likely dominated by a self-absorbed jet component (see Tinti et al. 2005). Flux density and spectral shape variability is an important tool to make a proper classification, but it needs to be complemented by an accurate pcscale radio morphological classification. Intrinsically small and young radio sources are expected to have a "Double/Triple" structure like in CSOs, while a Core-Jet morphology is more typical of blazar objects.

These two pieces of evidence need to be considered together.

Given the short time spent on each source, our VLBA observations have little or no sensitivity to complex structures covering a large number of pixels, but this should not play a major role for most of the sources discussed here. In the morphological classification given in Table 2, also the spectral information derived by comparing the flux densities of the components detected at two frequencies is very relevant. In general all the source components visible in Figs. 1 and 2 have steep spectra, while it is not the case for those in Figs. 3 and 4.

In Table 3 and Table 4 for each component of our CSO and Core-Jet candidate sources, we present the physical parameters. The total flux density and the Full Width Half Maximum (FWHM) have been obtained by fitting the components with a Gaussian model (task JMFIT).

Steep spectrum components are labelled as North (N), South (S), East (E), West (W), Central (Ce) depending on the source orientation and the component location, while a flat spectrum core is labelled $\mathrm{C}$, when detected. Beyond this, our core candidates must be unresolved.

\subsubsection{CSO morphology}

The sources discussed here have been observed in the optically thin part of their radio spectrum, but at frequencies at a factor of a few within their peak. In case of different component size and/or flux density within the same object, it is possible that the individual spectra of the two (or more) components, peak at different frequencies. Therefore, the observed spectral index in our data may be affected showing different values.

Fourteen sources ( 7 galaxies J0003+2129, J0037+0808, $\mathrm{J} 0428+3259, \quad \mathrm{~J} 1511+0518, \quad \mathrm{~J} 1735+5049, \quad \mathrm{~J} 1855+3742$ and J2203+1007, 5 quasars J0005+0524, J0650+6001, $\mathrm{J} 1148+5254, \mathrm{~J} 1335+4542$ and $\mathrm{J} 1616+0459$, and 2 empty field J0638+5933, J1335+5844) show a "Double/Triple" morphology, similar to those found in CSO sources (Figs. 1, 2). Ten sources have a Double structure characterised by two separated components where the flux density asymmetry is rather large, like in J0003+2129. Four sources are characterised by a Triple morphology (J0428+3259, J0650+6001, $\mathrm{J} 1511+0518$ and $\mathrm{J} 2203+1007$ ), where all the components have a steep spectrum. Since the presented data do not have either the dynamic range or the resolution suitable to detect weak flat components, there is no secure core identification so far. The source $\mathrm{J} 1855+3742$ is the only one which shows a quite complex structure with steep spectrum.

Their optically thin spectral index is much steeper than 0.5 . Only the sources J0638+5933, J1335+5844 and J1735+5049 
have spectral indices of $\sim 0.5$ or little flatter. Despite Peck \& Taylor (2000) did not consider J1335+5844 and J1735+5049 CSO candidates, not including them in the COINS sample, we still classify them as young object candidates. For J1335+5844, the source core has been identified, using high dynamic range VLBI images (Dallacasa et al. 2005), and this object can be considered a case study.

All these sources were not found to be variable in multiepoch VLA observations, and they are characterised by low value of the variability index $V$, as defined by Tinti et al. (2005). Two exceptions are J0650+6001, which shows a little variability in its flux density at high frequencies, and $\mathrm{J} 1616+0459$ where the flux density has decreased at all frequencies, but keeping the spectral shape unchanged. For the source $\mathrm{J} 1148+5254$, only a single-epoch VLA observation is available, and then it is not possible to assess its variability.

The source J0428+3259 is the only object which shows some extended emission on the arcsecond-scale (Tinti et al. 2005), although such extension could not be properly imaged in a relatively deep $L$ band image.

\subsubsection{Core-Jet morphology}

In this sub-section will be discussed objects not considered genuine young radio sources anymore. Six objects (five quasars J0329+3510, J2021+0515, J2114+2832, J2123+0535, J2136+0041 and one BL Lac J1811+1704), show a Core-Jet morphology, where one compact and bright component with a quite flat or inverted spectrum dominates the radio emission (from the $70 \%$ up to more than $90 \%$ of the total flux density of the source). On the other hand, the jet component accounts only for a little percentage of the total flux density and generally has a steeper spectrum.

Three sources (J0329+3510, J1811+1704 and 2123+0535) did not show the convex form in the second epoch spectrum (Tinti et al. 2005), which actually turns out to be flat. Therefore, these sources are classified as blazar and they will not be considered HFP candidates anymore. All these three blazar sources also show some extended emission on the arcsecondscale (Tinti et al. 2005).

The Core and Jet components are also characterised by very different physical parameters: high magnetic field and small emitting area for the Core component, while lower magnetic fields, more similar to those found in CSO components, and a larger emitting area for the Jet structure. These properties will be discussed in more detail in Sect. 5.3.

\subsection{Radio structure and optical identification}

There is a clear segregation in radio morphology between quasars and galaxies. The majority of galaxies $(\sim 78 \%)$ show a "Double/Triple" morphology while quasars are generally either Core-Jet $(\sim 16 \%)$ or Unresolved $(\sim 71 \%)$.

This is consistent with the idea that the HFP spectrum in galaxies and quasars appears originated from intrinsically different emitting regions: mini-lobes and/or hot-spots in galaxies, compact regions related to the core and to the jet base in quasars. This is in agreement with other results obtained by comparing the properties of galaxies and quasars in GPS and bright CSS samples (Stanghellini et al. 2005; Fanti et al. 1990). It is likely that HFP quasars (as well as GPS quasars) are intrinsically similar to the flat spectrum radio sources, and their convex radio spectrum is due to a single homogeneous component, like a knot in a jet, which dominates the radio emission, although some HFP quasars can still be genuine young radio sources.

Strong support to the idea that a significant fraction of HFP quasars represent a different population of beamed radio sources like blazars, comes from the detection of substantial variability of their radio spectra (Tinti et al. 2005). Indeed, during a second epoch of simultaneous multi-frequency VLA observations, carried out for monitoring the flux density, seven objects no longer exhibit the convex spectrum, which actually turned out to be flat. All these sources are associated with quasars with "Core-Jet", "Unresolved" or "Marginally Resolved" radio structure in our VLBA images, suggesting that they are blazars previously selected during a flare of a selfabsorbed component.

\subsection{Physical parameters}

Physical parameters for the radio sources have been computed assuming equipartition and using standard formulae (Pacholczyk 1970). Proton and electron energies have been assumed equal, with a filling factor of unity; ellipsoidal geometry and an average optically thin spectral index of 0.7 have been adopted. The average luminosity of the sources is $P \sim 10^{27.5} \mathrm{~W} / \mathrm{Hz}$ with a clear influence of redshift, being the most distant sources (quasars) the most luminous as well (Fig. 7). In the components of candidate CSOs, typical values obtained for minimum total energy $\left(U_{\min }\right)$, minimum energy density $\left(u_{\mathrm{min}}\right)$, equipartition magnetic field $\left(H_{\mathrm{eq}}\right)$, and brightness temperature $\left(T_{\mathrm{b}}\right)$ are:

$\left(U_{\min }\right) \sim 10^{53}-10^{54} \mathrm{erg}$

$\left(u_{\min }\right) \sim 10^{-4}-10^{-5} \mathrm{erg} \mathrm{cm}^{-3}$;

$\left(H_{\mathrm{eq}}\right) \sim 10^{-2} \mathrm{G}$;

$\left(T_{\mathrm{b}}\right) \sim 10^{8}-10^{11} \mathrm{~K}$.

The same values are found also in the jets of the HFPs with a Core-Jet morphology, while the core components are generally characterised by higher energy density and brightness temperatures, as they have larger luminosities and smaller sizes.

For comparison, the minimum energies stored in CSS radio sources (Fanti et al. 1990) of similar radio power are 2-3 order of magnitude larger and the corresponding energy densities are 2-3 order of magnitude lower.

In Fig. 8 we plot the magnetic field versus the emitting area. There is a clear segregation among the different morphologies. The bottom left part of the plot is dominated by CSO and jet components, while the core components and the unresolved sources are located in the upper part. This relation may be very useful to better classify CSO candidate. Indeed, as Core and Jet structures have deep differences in their equipartition magnetic 


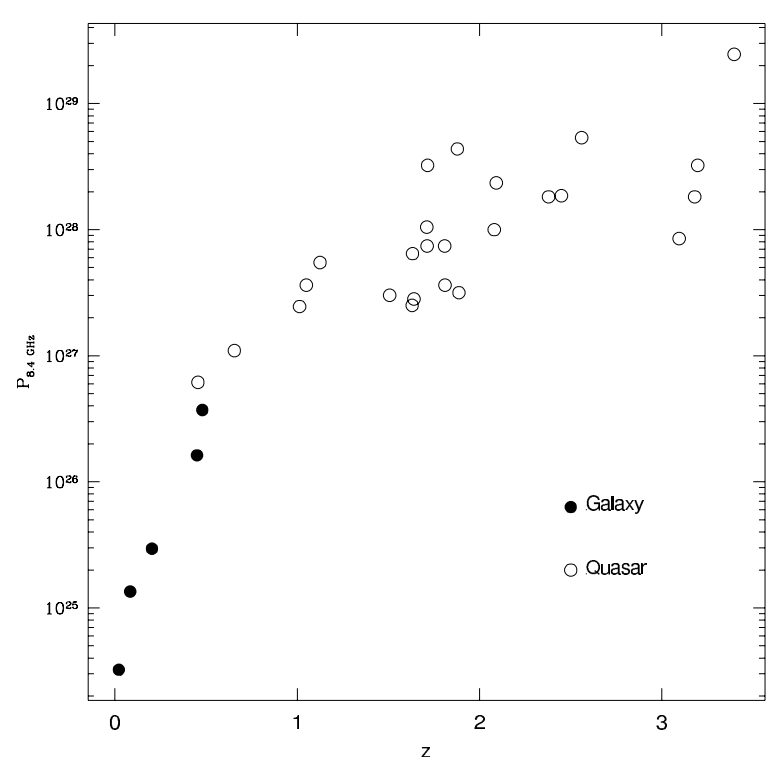

Fig. 7. Distribution of the monochromatic luminosity at $8.4 \mathrm{GHz}$ $(\mathrm{W} / \mathrm{Hz})$ versus the redshift, for the HFP objects with known $z$. The apparent luminosity has been computed on the basis of the VLA flux density. There is a clear influence of the redshift: galaxies, characterised by low redshift, are located in the bottom right part of the plot, while quasars dominate the upper left region.

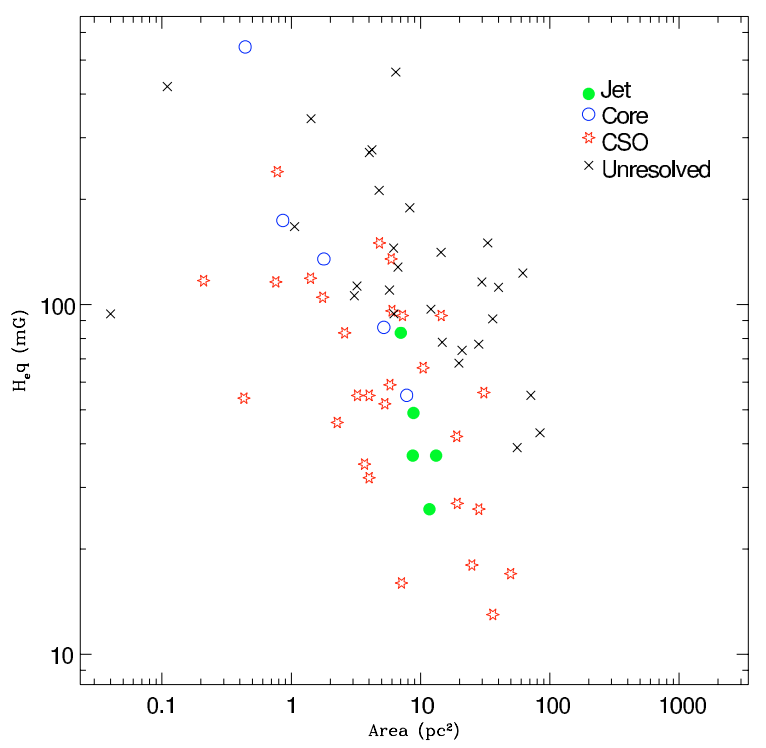

Fig. 8. Distribution of the emitting area versus the equipartition magnetic field.

field and emitting area, if we find such a trend also in a CSO candidate, it may suggest a possible wrong classification.

Following the definition by Readhead (1994), we computed the "equipartition brightness temperature". Since it depends mainly on the redshift and only very weakly on observed parameters, like the observed peak frequency and the flux density, it can be determined to high precision, becoming a good observable quantity against which to test theoretical models. The values we obtain range from $7 \times 10^{10} \mathrm{~K}$, up to $2.5 \times 10^{11} \mathrm{~K}$, in agreement with what found by Readhead (1994).

Moreover, assuming the turnover frequency from Dallacasa et al. (2000) and the equipartition magnetic field, we compute the maximum brightness temperature $T_{\mathrm{b} \text {,max }}$. The comparison between the $T_{\text {eq }}$ and $T_{\mathrm{b}, \max }$ is an important tool to investigate a possible departure from the minimum energy and equipartition condition in these object, since $T_{\mathrm{b}, \max } / T_{\mathrm{eq}}$ is an independent way to derive the equipartition Doppler factor (Readhead 1994). Therefore, a $T_{\mathrm{b}, \max } \gg T_{\mathrm{eq}}$ is a strong indication that that source is a blazar.

For all the unresolved sources, the core components and few of the brightest CSO components, we find that $T_{\mathrm{b}, \text { max }} / T_{\text {eq }} \sim$ $2-3$, while for a good fraction of CSO and jet components, it is $\leq 1$, indicating that in these structures the total energy is not far from the minimum energy condition.

\section{Summary}

We have presented the results of new VLBA observations at two different frequencies in the optically thin part of the spectrum for 51 HFPs from the "bright HFP sample" (Dallacasa et al. 2000). The conclusion from this investigation can be summarised as follows:

a) Morphology:

- fourteen sources $(27 \%)$ show a CSO-like morphology in our VLBA images;

- six sources (12\%) have a Core-Jet morphology;

- thirty-one sources (61\%) are Unresolved or Marginally resolved even at the VLBA resolution.

b) Optical identification related evidence:

- seven sources all identified with quasar (3 with a Core-Jet structure and 4 Unresolved) have been rejected, since their radio spectrum turned out to be flat;

- HFP galaxies and quasars have different morphological properties. The $78 \%$ of the galaxies show a CSO-like morphology, while quasars have Core-Jet (16\%) or Unresolved (71\%) morphology.

c) relationship with flux density variability:

- three Unresolved and Marginally Resolved sources (2 quasars and $1 \mathrm{BL}$ Lac), show substantial flux density variability, and they are not considered HFP candidates anymore;

- generally galaxies do not show any evidence of variability.

In general, the pc-scale information available from these short VLBA observations and low-dynamic range images, becomes more effective if complemented with observations of the flux density and spectral shape variability.

Milli-arcsecond images with high dynamic range, polarisation measurements, together with a multi-epoch flux density monitoring can help to find other contaminating objects of the original sample. The analysis of a third epoch of simultaneous multi-frequency VLA observations and polarisation data is in progress. At the end, we will be able to construct a sample of confirmed genuine and young HFP radio sources. 
Acknowledgements. The VLBA is operated by the US National Radio Astronomy Observatory which is a facility of the National Science Foundation operated under a cooperative agreement by Associated Universities, Inc. This work has made use of the NASA/IPAC Extragalactic Database NED which is operated by the JPL, California Institute of Technology, under contract with the National Aeronautics and Space Administration.

\section{References}

Bicknell, G. V., Dopita, M. A., \& O’Dea, C. P. O. 1997, ApJ, 485, 112 Chavushyan, V., Mujica, R., Gorshkov, A. G., et al. 2001, ARep, 45, 79

Condon, J. J., Cotton, W. D., Greisen, E. W., et al. 1998, AJ, 115, 1693

Dallacasa, D., Stanghellini, C., Centonza, M., \& Fanti, R. 2000, A\&A, 363,887

Dallacasa, D., Falomo, R., \& Stanghellini, C. 2002, A\&A, 382, 53

Dallacasa, D. 2003, PASA, 20, 79

Dallacasa, D., Venturi, T., Stanghellini, C., et al. 2005, MemSAIt, 76,134

Dallacasa, D., Falomo, R., \& Stanghellini, C. 2006, A\&A, in preparation

Edwards, P. G., \& Tingay, S. J. 2004, A\&A, 424, 91

Fanti, R., Fanti, C., Schilizzi, R. T., et al. 1990, A\&A, 231, 333

Fanti, C., Fanti, R., Dallacasa, D., et al. 1995, A\&A, 302, 317

Gregory, P. C., Scott, W. K., Douglas, K., \& Condon, J. J. 1996, ApJS, 103,427

Gugliucci, N. E., Taylor, G. B., Peck, A. B., \& Giroletti, M. 2005, ApJ, 622, 136

Kudnic, T., Hogg, D. W., Blandford, R. D., et al. 1997, AJ, 114, 2276

Murgia, M. 2003, PASA, 20, 19
O’Dea, C. P. 1998, PASP, 110, 493

Pacholczyk, A. G. 1970, Radio Astrophysics (San Francisco: Freeman \& Co.)

Patnaik, A. R., Browne, I. W. A., Walsh, D., et al. 1992, MNRAS, 259, 1

Patnaik, A. R., Kemball, A. J., Porcas, R. W., \& Garrett, M. A 1999, MNRAS, 307, 1

Peck, A. B., \& Taylor, G. B. 2000, ApJ, 534, 90

Polatidis, A. G., Wilkinson, P. N., Xu, W., et al. 1999, NewAR, 43, 657

Polatidis, A. G., \& Conway, J. E. 2003, PASA, 20, 69

Readhead, A. C. S. 1994, ApJ, 426, 51

Readhead, A. C. S., Taylor, G. B., Xu, W., et al. 1996, ApJ, 460, 612

Shimmins, A. J., Searle, L., Andrew, B. H., \& Brandie, G. W. 1968, ApJ, 1167

Snellen, I. A. G., Schilizzi, R. T., Miley, G. K., et al. 2000, MNRAS, 319,445

Stanghellini, C., O’Dea, C. P. O., Dallacasa, D., et al. 1998, A\&AS, 131,303

Stanghellini, C., O’Dea, C. P., \& Murphy, D. W. 1999, A\&A, 134, 309

Stanghellini, C., O'Dea, C. P., Dallacasa, D., et al. 2005, A\&A, 443, 891

Stickel, M., \& Kühr, H. 1996, A\&AS, 115, 11

Tinti, S., Dallacasa, D., de Zotti, G., Celotti, A., \& Stanghellini, C. 2005, A\&A, 432, 31

Tornikoski, M., Jussila, I., Johansson, P., Lainela, M., \& Valtaoja, E. 2001, AJ, 121, 1306

Xiang, L., Stanghellini, C., Dallacasa, D., \& Haiyan, Z. 2002, A\&A, 385,768

Wilkinson, P. N., Polatidis, A. G., Readhead, A. C. S., Xu, W., \& Pearson, T. J. 1994, ApJ, 432, L87 


\section{Online Material}


M. Orienti et al.: VLBA images of high frequency peakers, Online Material p 2
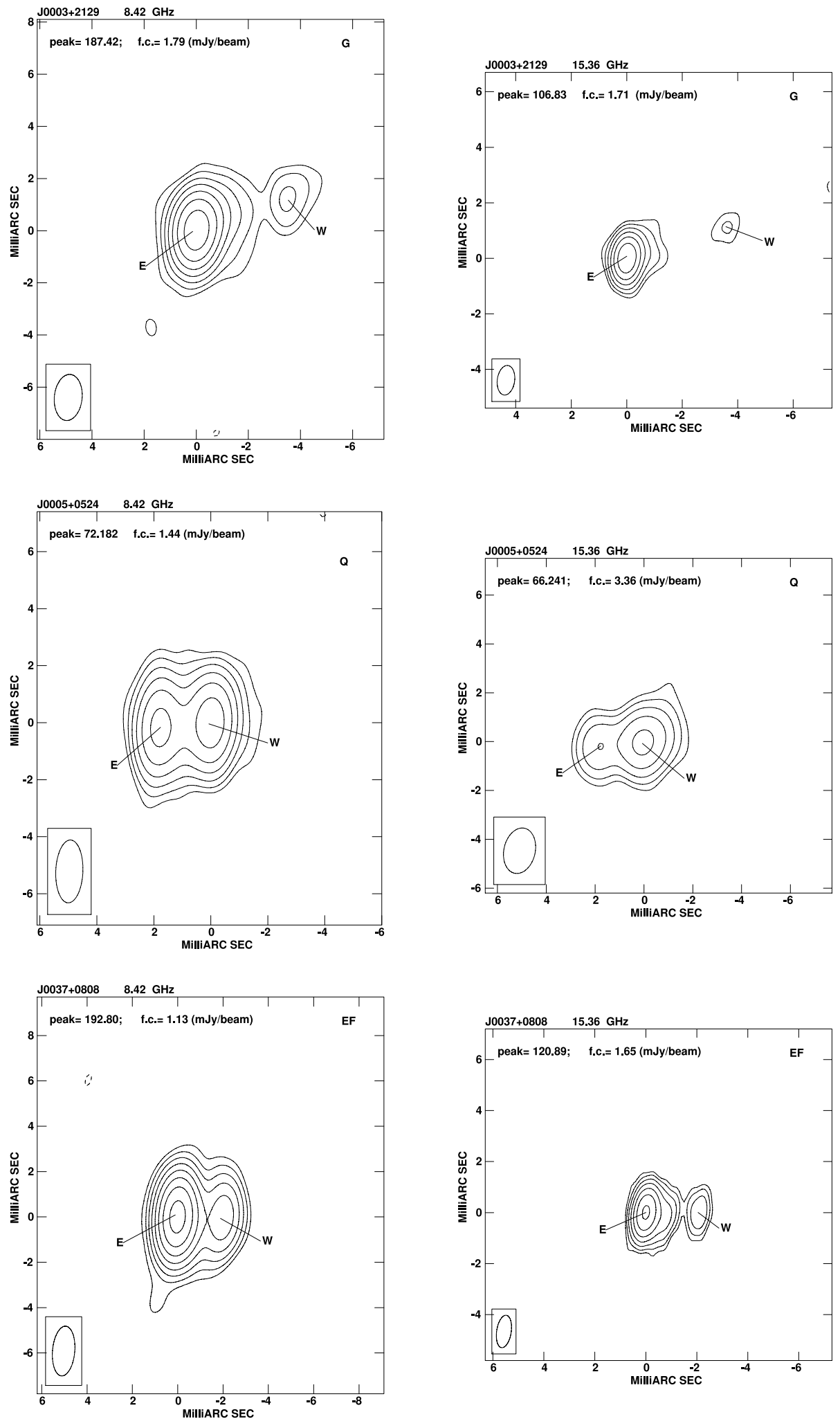

Fig. 1. VLBA images at the two frequencies of the candidate HFPs with a CSO morphology. For each image we give the following information on the plot itself: a) peak flux density in $\mathrm{mJy} /$ beam; b) first contour intensity (f.c., in $\mathrm{mJy} /$ beam), which is generally 3 times the rms noise on the image plane; contour levels increase by a factor of 2; c) the optical identification; d) the restoring beam is plotted on the bottom left corner of each image. 
M. Orienti et al.: VLBA images of high frequency peakers, Online Material p 3
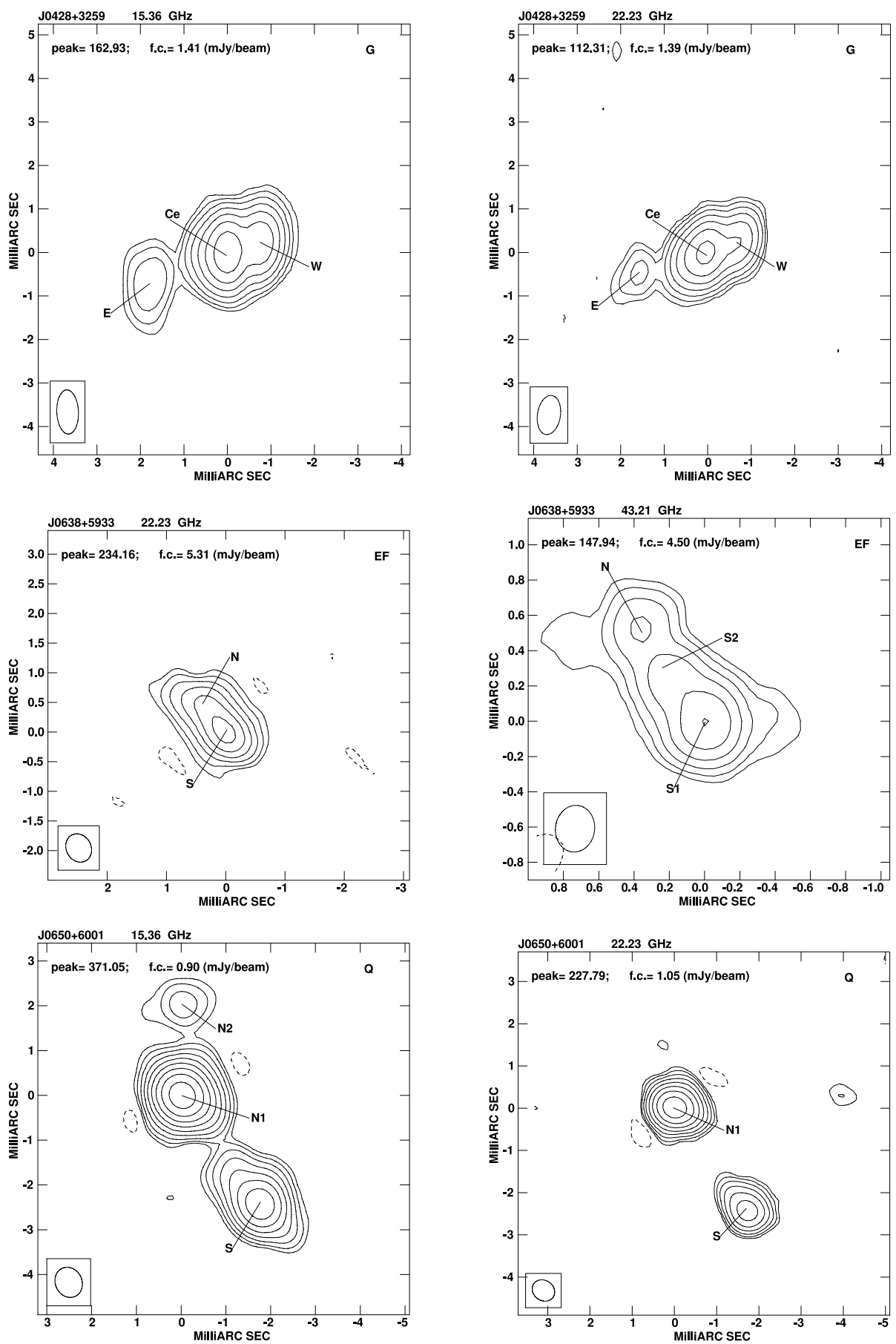

Fig. 1. continued. 
M. Orienti et al.: VLBA images of high frequency peakers, Online Material p 4
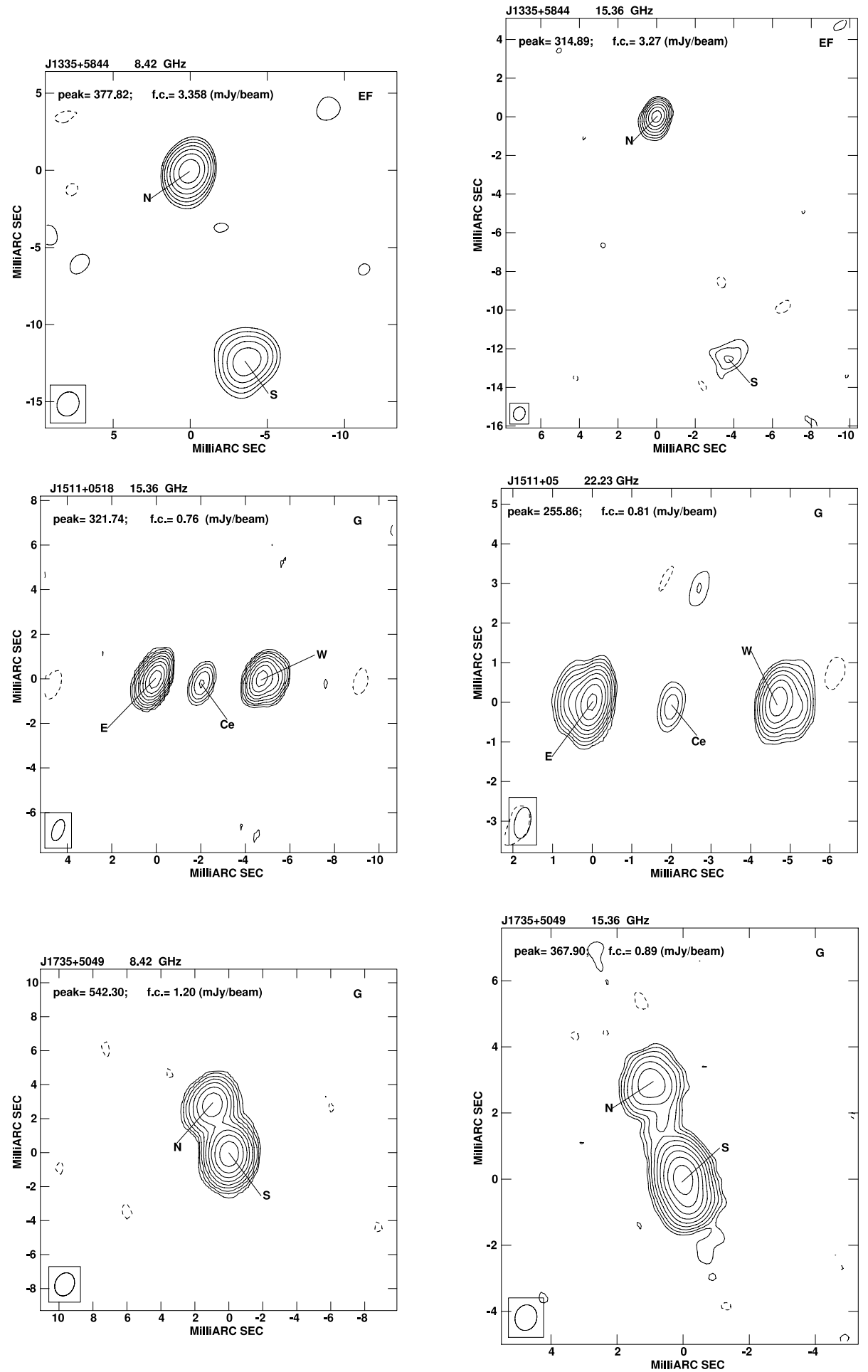

Fig. 1. continued. 
M. Orienti et al.: VLBA images of high frequency peakers, Online Material p 5
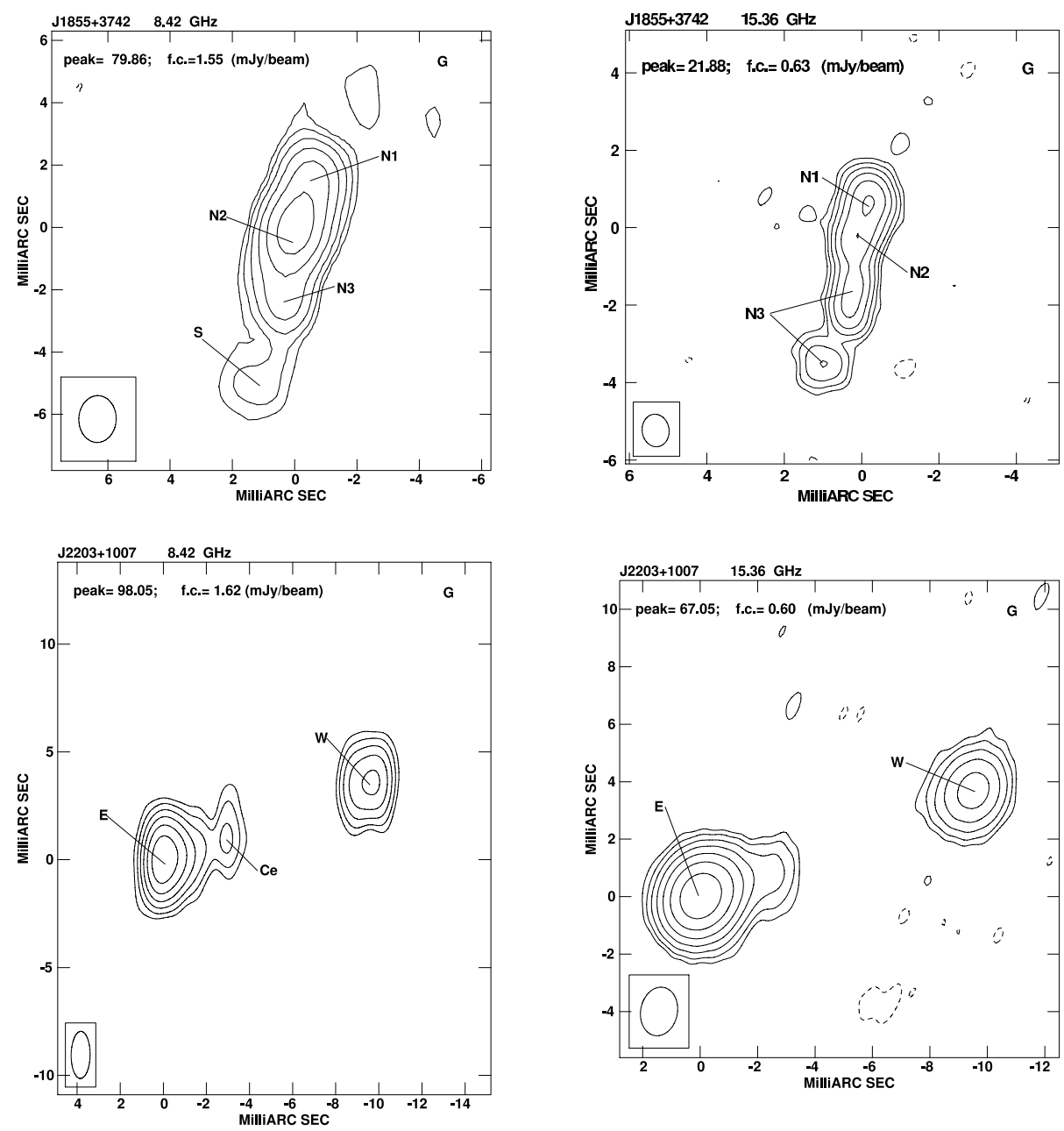

Fig. 1. continued. 